\title{
Microbiology of chronic suppurative otitis media,study of the role of bacterial biofilm and fungal infection
}

\begin{abstract}
Chronic suppurative otitis media (CSOM) is defined as chronic inflammation of middle ear cleft. The disease is common in all age groups but more prevalent in low socioeconomic group. It is most commonly seen in developing and undeveloped countries affecting 0.5 $30 \%$ of any community. Local application of antibiotics remains important and first step to get a dry ear. Changes in bacterial flora in last decade and abuse of antibiotics lead to the emergence of multi-drug resistant organisms. Recently, bacterial biofilm are thought to have a major role in much otolaryngologic infection. The formation of biofilm facilitates chronic bacterial infections and reduce efficacy of anti-microbial therapy. It is estimated that biofilm account of approximately $60 \%$ of microbial infection in the body. In addition to bacterial biofilm, the role of fungal infections in CSOM needs more attention. Fungal infections of middle ear are common as fungi thrive well in moist pus. There is very little known about mycological aespect of these ears, the importance of which has increasing in the recent years because of the excessive use of broad sepectrum antidiotics, corticosteroids and cytotoxic chemotherapy.
\end{abstract}

Aim: Microbiological examination of chronic suppurative otitis media by detecting bacterial and fungal isolates involved in the infection. Additionally, the detection of the capacity of bacterial biofilm formation.

Patients and methods: Forty Patients suffering from chronic suppurative otitis media attending outpatient clinic in Menoufia university hospital and Karmoz insurance hospital were included in this study. Pus discharge was collected using sterile cotton swabs. These samples were cultured for detection of bacterial and fungal infections and Detection of bacterial capability of forming biofilm was done using crystal violet method and by Overnight culture in tryptic Soya Broth (TSB).

Results: Most common isolated bacteria were pseudomonas aeurgiosa (37.5\%), followed by Klebseilla.spp (10\%) and Staph.aeureus $(7.5 \%)$ and fungal culture gave results to Candida (10\%) and Asperigillus (7.5\%). Mixed bacterial and fungal infection occurred in (11.7\%) of our patients.14 out of 15 cases of pseudomonas infection gave results to biofilm formation(93.3\%)and Staph.aeureus infection, 3 cases out of 3 cases(100\%) were biofilm forming.

Conclusion: Routine culture and sensitivity remains the most important step in management of CSOM because of its multi-etiology that makes the empirical therapy impossible. Fungal infection can occur without clinical evidence, therefore, empirical local antifungal therapy is strongly recommended in such cases. Biofilm formation may be responsible for irresponsiveness to antibiotic chemotherapy. Physical removal or inhibition of biofilm may enhance the response to antimicrobial therapy and infection eradication.

Keywords: chronic suppurative otitis media (csom), bacterial biofilm, fungal infection
Volume 3 Issue I - 2015

\author{
Ibrahim A Abdelshafy,' Ahmed A Haleem,' \\ Yasser A Khalil,' Abeer A Ghazal, ${ }^{2}$ Ahmed \\ Gaballah, ${ }^{2}$ Ahmed Saied ${ }^{3}$ \\ 'Department of Otorhinolaryngology, Menofya University, Egypt \\ 2Department of Microbiology, Alexandria University, Egypt \\ ${ }^{3}$ Department of Otorhinolaryngology, Karmouz Health \\ Insurance Hospital, Egypt
}

Correspondence: Ibrahim A Abdelshafy, Department of Otorhinolaryngology, Menoufia Faculty of Medicine,Yassin Abdel-Ghaffar St., Shebein Elkom, Egypt, Tel 002010028I26I2, Email Ibrahim_ent@yahoo.com

Received: May 24, 2015 | Published: August 4, 2015
Abbreviations: CSOM, chronic suppurative otitis media; ABR, antibiotic resistance; TSB, tryptic soya broth; CLSI, clinical and laboratory standard institute; OD, optical density

\section{Introduction}

Chronic suppurative otitis media (CSOM) is defined as chronic suppurative inflammation of muco periosteal lining of middle ear cleft with tympanic membrane perforation. ${ }^{1,2}$ The disease is common in all age groups but more prevalent in low socioeconomic group. It is most commonly seen in developing and undeveloped countries affecting $0.5-30 \%$ of any community. ${ }^{3}$ CSOM has profound impact on society in terms of hearing of patient. ${ }^{4}$ It causes conductive and sensorineural hearing loss and adverse effects on child development. ${ }^{5}$
CSOM is of two types, mucosal or tub tympanic type and cholesteatoma or atticoantral type. ${ }^{6}$ Tub tympanic type affects mainly mesotympanum, hypo tympanum, Eustachian tube and mastoid air cells where as atticoantral is a dangerous one because of associated complications and may be life threatening at times infection can spread from middle ear to vital structures as mastoid, facial nerve, labyrinth, lateral sinus, meninges and brain leading to mastoid abscess, facial nerve paralysis, deafness, lateral sinus thrombosis, meningitis and brain abscess. ${ }^{7}$ Of all these complications, hearing loss associated with chronic ear discharge is almost reported in $50 \%$ of cases. ${ }^{8}$ Changes in bacterial flora in last decade and abuse of antibiotics lead to multidrug resistant organisms. There is a major gap in knowledge about the magnitude of this problem and such information is needed to guide urgent public health actions. Antibiotic Resistance (ABR) is complex 
and multidimensional. It involves a range of resistance mechanisms producing an over - widening range of bacteria, most of which can cause a wide spectrum of diseases in humans and animals. ${ }^{9}$

Recently, bacterial biofilm are thought to have a major role in many otolaryngologic infection, Over the past 20 years a new appreciation has developed regarding how bacteria behave differently once bound to a surface..$^{10}$ Biofilms are complex and organized bacterial communities that are able to grow in association with different biological or inert surfaces. Surface bound bacteria grow into biofilm, which are colonies of slowly growing bacteria that surround themselves in a coat of glycol polysaccharides called a glycol calyx. ${ }^{11}$ The clinical consequence of the biofilm mode of growth relates to the difficulty of therapeutic eradication of sessile cells such supra-cellular structure. The formation of biofilm facilitates chronic bacterial infections and reduce efficacy of anti-microbial therapy. ${ }^{12,13}$ It is estimated that biofilm account of approximately $60 \%$ of microbial infection in the body. In addition to bacterial biofilm, role of fungal infections in CSOM needs more attention. Fungal infections of middle ear are common as fungi thrive well in moist pus. ${ }^{14}$ There is very little known about mycological aspect of these ears, the importance of which has increasing in the recent years because of the excessive use of broad spectrum antibiotics, corticosteroids, cytotoxic chemotherapy and increase in number of immune deficiency conditions.

\section{Patients and methods}

Forty Patients suffering from CSOM attending outpatient clinic in Menoufia University Hospital and Karmoz Insurance hospital were included in this study. Patients receiving antibiotics $48 \mathrm{hrs}$ prior to sample collection, patient with clinical evidence of fungal infection; presented with dots and/or fungal hyphae, as well as patients with mixed otitis media and otitis externa infections were excluded from this study. ${ }^{15}$

\section{Materials and methods}

Otherwise stated, all microbiological media and reagents were supplied by Oxoid LTD. (Hamphshire, England).

\section{Collection and transport of specimens}

Three swabs were collected by sterile cotton swab from each patient. Swabs were transported directly to the microbiology laboratory at the Medical Research Institute, Alexandria University. If immediate transportation was not possible, one of the swabs was dipped in Amies transport media and stored till next day at room temperature.

\section{Direct visual examination}

One swab was used to prepare a direct smear of the specimen. The smear was Gram stained and examined for the presence of pus cells, bacteria and/or fungal elements.

\section{Culture}

For bacterial cultures, all specimens were inoculated on blood, and MacConkey's agar plates. For fungal culture, specimens were also inoculated on Sabouraud dextrose agar plates. Plates were incubated overnight at $37^{\circ} \mathrm{C}$. Sabouraud dextrose plates that did not show growth were kept for 5 days at room temperature before being discarded. Bacterial growth was identified using colonial morphology, Gram stain results and key biochemical reactions. All bacterial isolates were subjected to antimicrobial susceptibility testing using Kirby-Bauer method using selected antibiotic panel according to Clinical and Laboratory Standard Institute (CLSI). ${ }^{16}$

\section{Quantitative determination of biofilm formation}

This method was performed to all isolated bacterial pathogen and candida spp. Overnight culture in tryptic Soya Broth (TSB) were dilute 1:100 with fresh TSB. Three wells of a sterile 96-well flat bottomed plastic tissue culture plate with a lid (Cellstar, greiner bioone) were filled with $0.2 \mathrm{ml}$ of each bacterial suspension. A negative control was used containing sterile broth without bacteria.

The plates were covered and incubated aerobically for 24 hours at $37^{\circ} \mathrm{C}$. After incubation, the content of the wells was aspirated and each well was washed 3 times with $0.25 \mathrm{ml}$ sterile physiological saline. The plate was vigorously shaken to remove all non-adherent bacteria. The remaining attached bacteria were fixed using $0.2 \mathrm{ml}$ of absolute methanol per well for 15 minutes followed by methanol removal and air drying. The plates were stained for 5 minutes with $0.2 \mathrm{ml}$ of $0.2 \%$ Hucker crystal violet solution. Excess stain was rinsed with running tap water. The plates are allowed to air dry (Table 1).

Table I Quantitative determination of biofilm formation

\begin{tabular}{|c|c|c|c|c|c|}
\hline & Non Adherent & Weakly Adherent & Moderately Adherent & Strongly Adherent & Total \\
\hline Pseudomonas Aeruginosa & 0 & 6 & 8 & 1 & 15 \\
\hline Klebsiella Spp & I & 0 & 3 & 0 & 4 \\
\hline Staphylococcus Aureus & 0 & 0 & 2 & I & 3 \\
\hline Diphtheroids & I & 2 & 0 & 0 & 3 \\
\hline Acinetobacter Baumannii & 0 & 2 & I & 0 & 3 \\
\hline Enterobacter Spp & 0 & 0 & I & 0 & I \\
\hline E. Coli & 0 & I & 0 & 0 & I \\
\hline Candida Albicans & 3 & 2 & 0 & 0 & 5 \\
\hline Total & 5 & 13 & 15 & 2 & 35 \\
\hline
\end{tabular}

The dye bound to the adherent cells was resolubilized with $160 \mu 1$ of $30 \%(\mathrm{v} / \mathrm{v})$ glacial acetic acid. The optical density (OD) of each well was measured at $590 \mathrm{~nm}$ using automated plate reader. The cutoff OD (ODc) was calculated as three standard deviations above the mean OD of the negative control wells. Bacterial isolates showing mean OD less than ODc were considered non adherent. On the other hand bacterial isolates showing mean OD more than ODc, more than 2times ODc and more than 3times ODc were considered weakly adherent, moderately adherent and strong adherent respectively.

\section{Results}

In the present study, 26 cases (65\%) showed single bacterial pathogen, 3 cases $(7.5 \%)$ showed two bacterial pathogens, 4 
cases $(10 \%)$ showed mixed bacterial and fungal infections and 1 case $(2.5 \%)$ showed mixed fungal infection with Aspergillus spp and Candida albicans. On the other hand, 6 cases (15\%) did not show bacterial or fungal growth (Figure 1). Bacterial and fungal identification revealed that Pseudomonas aeruginosa was the most commonly isolated pathogen (15 cases) among our 40 CSOM cases followed by Klebsiella spp (4 cases), Staphylococcus aureus (3 cases), Acinetobacter baumannii (3 cases), Diphtheroids (3 cases) and E. coli (1 case) and Enterobacter spp (1 case). Candida albicans was the most commonly isolated fungal pathogen (6 cases) followed by Asperigallus flavus (4 cases) (Figure 2). Using direct examination of Gram stained film of the specimen enabled the detection of fungal hyphae in 4 cases in which Asperigallus flavus were isolated from the fungal culture. The antimicrobial susceptibility testing of Pseudomonas aeruginosa and Staphylococcus aureus are shown in Figure 3 \& 4.

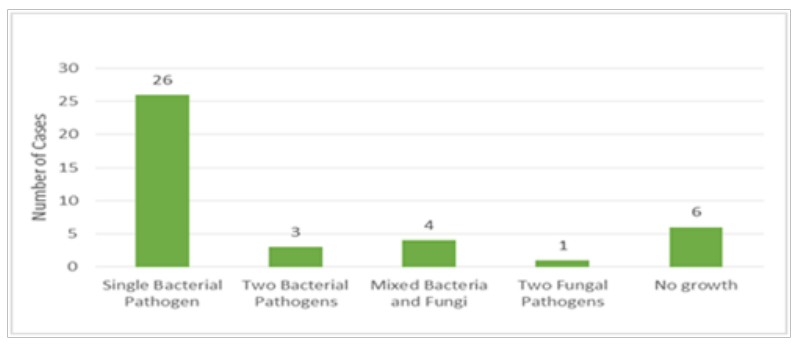

Figure I Bacterial and fungal culture results.

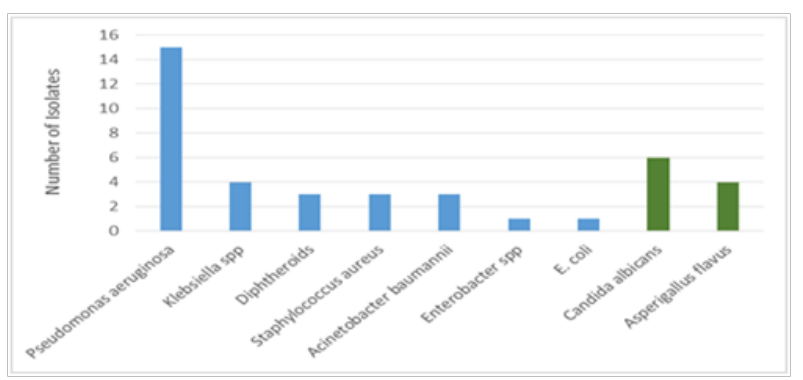

Figure 2 Bacterial and fungal isolates among CSOM cases.

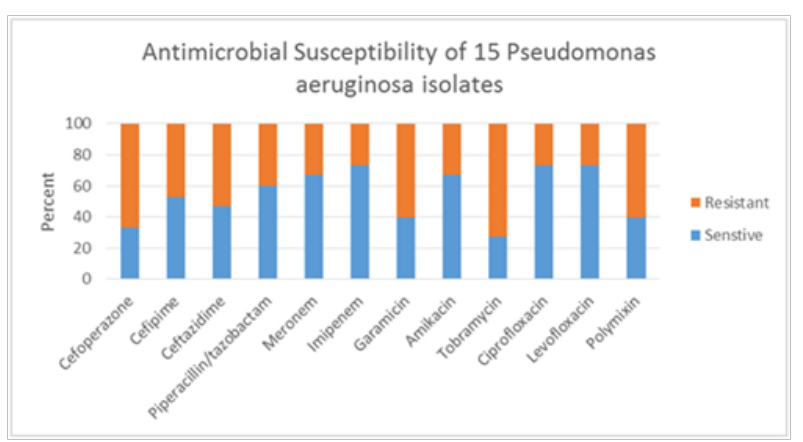

Figure 3 The antimicrobial susceptibility testing of Pseudomonas aeruginosa.

\section{Biofilm formation}

Out of 35 isolated bacterial and candida isolates, 30 isolates $(85.7 \%)$ of them were capable of biofilm formation Where 15 isolates were moderately adherent, 13 were weakly adherent and only 2 were strongly adherent. Concerning Psuedomonas aeruginosa and Staphylococcus aureus, the most commonly isolated Gram negative and positive isolates respectively, all isolates showed biofilm formation capability. All S. aureus isolates as well as $60 \%$ of Peudomonas isolates were moderately and strongly adherent. Only 2 isolates of Candida albicans (40\%) were weakly adherent, suggesting weaker capability of biofilm formation in comparison with bacterial isolates (Table 1).

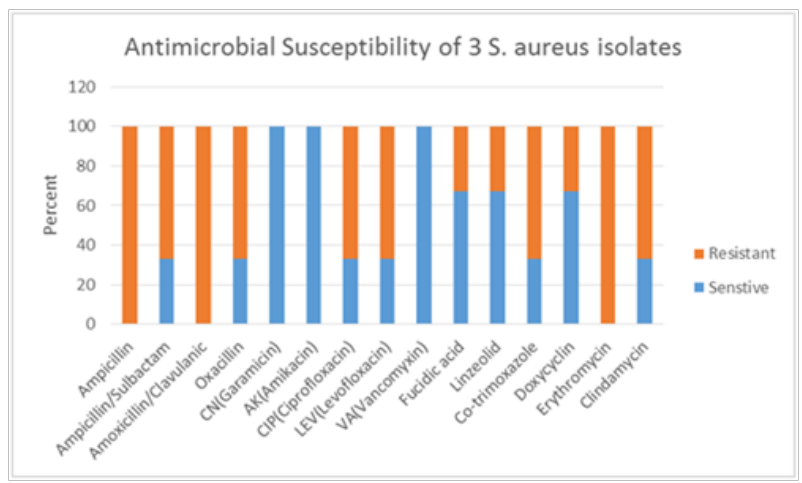

Figure $\mathbf{4}$ The antimicrobial susceptibility testing of Staphylococcus aureus.

\section{Discussion}

Chronic suppurative otitis media (CSOM) is persistent inflammation of the middle ear or mastoid cavity. it usually begins as a complication of persistent acute otitis media (AOM) with perforation in childhood. Typical findings may also include thickened granular middle ear mucosa and mucosal polyps. ${ }^{17}$ The worldwide prevalence of CSOM is 65 to 330 million persons, and 39 to 200 million (60\%) of those individuals has clinically significant hearing impairment. ${ }^{17}$

The definitive treatment of CSOM is by surgery (tympanoplasty and/or mastoidectomy), nevertheless, initial treatment by ear toilet and ototopical agents is necessary to prepare the ear for surgery. The selection of local or systemic antibiotic for therapy depends greatly on the type of the organism isolated in such cases. In the present study, Pseudomonas aeruginosa was the most commonly isolated bacterial pathogen followed by Klebsiella, Staphylococci, Diphtheroids and Acinetobacter. Additionally, mixed bacterial infection was detected in 3 out of 40 cases included in this study. Analysis of the antimicrobial susceptibility results showed that the aminoglycoside Amikacin had 67\% and 100\% efficiency against Pseudomonas aeruginosa and Staphylococcus aureus supporting its selection as empirical therapy in CSOM. This finding is in concordance with previous results reported from India, Nigeria and Nepal.

On the other hands fluoroguinolones such as ciprofloxacin and levofloxacin that showed $73 \%$ against Pseudomonas aeruginosa, showed only 33\% efficacy with Staphylococcus isolates. Carbapenem antibiotics, imipenem and meropenem, showed 100\% against Gram negative pathogens including Psuedomonas aeruginosa. On the other hand, with high incidence of community acquired methicillin resistant Staphylococcus aureus, the use of beta-lactam antibiotics as an empirical therapy is not recommended when $S$. aureus infection is suspected..$^{18}$ In our study 2 out of 3 Staphylococcus aureus isolates were methicillin resistant. The second predominant organism in our study was Staphylococcus aureus which is similar to findings of various previously conducted studies in the world and often follows pseudomonas infection. ${ }^{19}$

In the present times the indiscriminate use of broad spectrum antibiotics, corticosteroids, cytotoxic chemotherapy as well as the increased incidence of conditions accounting for immune deficiency has led to the increase prevalence of superadded fungal infection in CSOM. It has been suggested that the irrational and excessive use of 
topical antibiotic and steroids for all cases with COM, promotes the growth of Fungus. ${ }^{20}$ In the present study, co-infection with fungi was detected in $10(25 \%)$ out of 40 CSOM cases. Additionally Candida with Aspergillus flavus were the only isolated pathogens in one of the cases. In all previously mentioned cases, no evidence of fungal infection could be detected upon clinical examination. This finding makes the laboratory investigation for fungal infections in CSOM cases indispensable. The co-administration of topical antifungal with antibacterial agent is greatly recommended.

Despite of antimicrobial susceptibility testing, it was noticed clinically that 4 of our cases did not show response to suggested antibiotic therapy. Biofilm formation could be greatly accused for the irresponsiveness of CSOM cases to antibiotic therapy. In biofilms, poor antibiotic penetration, nutrient limitation, slow growth, adaptive stress responses, and formation of persistent cells are hypothesized to constitute a multi-layered defense. ${ }^{21}$ In the present study, 30 (85.7 $\%)$ of 35 bacterial and candida isolates showed adherence possibility to inanimate surfaces indicating biofilm formation capability. Three out of 4 non-responding cases were infected with Pseudomonas aeruginosa and one with $S$. aureus showing moderate to strong adherence capability.

Our study clearly indicate the importance of the human immune response to biofilm infections and suggest that in addition to the massive effort to discover novel anti-biofilm antibiotics, understanding the role of the immune system in biofilm infections is vital to reducing the burden of these infections. ${ }^{22}$ There are few researches regarding biofilm eradication, one of them showed that overnight incubation of lactoferrin reduce the biofilm activity of the $P$. aeruginosa isolates. In addition, it was reported that Lactoferrin is able to prevent biofilm formation. Nevertheless, the most successful procedure in biofilm forming infections is physical removal e.g. suction and aural toilet. ${ }^{23}$

\section{Conclusion}

Routine culture and sensitivity remains the most important step in treating CSOM because of its multi-etiology that makes the empirical therapy impossible. Fungal infection can occur without clinical evidence, therefore, empirical local antifungal therapy is strongly recommended in such cases. Biofilm formation may be responsible for irresponsiveness to antibiotic chemotherapy. Physical removal or inhibition of biofilm may enhance the response to antimicrobial therapy and infection eradication.

\section{Acknowledgments}

None.

\section{Conflicts of interest}

Author declares there are no conflicts of interest.

\section{Funding}

None.

\section{References}

1. Wintermeyer SM, Nahata MC. Chronic suppurative otitis media. Ann Pharmacothe. 1994;28(9):1089-1099.

2. Chronic suppurative Otitis media. Prodigy. 2008.
3. Adhikari P, Sinha BK, Pokhrel NR, et al. Prevalence of chronic suppurative otitis media in school children of kathmandu district. Journal of institute of medicine. 2007;29(3):10-12.

4. Dugdale AE. Management of chronic suppurative otitis media. Med J. 2004;180(2):91-93.

5. El-sayed Y. Bone conduction impairment in uncomplicated chronic suppurative otitis media. Am J Otolaryngol. 1998;19(3):149-153.

6. Kumar H, Seth S. Bacterial and fungal study of 100 cases of chronic suppurative otitis media. J Clin Diagn Res. 2011;5(6):1224-1227.

7. Rout MR, Mohanty D, Vijaylaxmi Y, et al. Prevalence of cholesteatoma in chronic suppurative otitis media with central perforation. Indian $J$ Otol. 2012;18(1):7-10.

8. Morris PS, Leach AJ, Silberberg P, et al. Prevention and management of chronic suppurative otitis media in aboriginal children a practical approach. Comm Ear Hearing H. 2007;4:22-25.

9. Harnivender Kumar, Sonia Seth. Bacterial and Fungal Study of 100 Cases of Chronic Suppurative Otitis Media. Journal of Clinical and Diagnostic Research (Suppl-1). 2011;5(6):1224-1227.

10. Costerton JW, Stewart PS, Greenberg EP. Bacterial biofilms: a common cause of persistent infections. Science. 1999;284(5418):1318-1322.

11. Costerton JW, Geesey GG, Cheng KJ. How bacteria stick. Sci Am. 1978;238(1):86-95.

12. Castillo MG, del Campo R, Baquero F, et al. Stationary biofilm growth normalizes mutation frequencies and mutant prevention concentrations in Pseudomonas aeruginosa from cystic fibrosis patients. Clin Microbiol Infect. 2011;17(5):704-711.

13. Stoodley HL, Stoodley P. Evolving concepts in biofilm infections. Cell Microbiol. 2009;11(7):1034-1043.

14. Ibekwe AO, al Shareef $\mathrm{Z}$, Benayam A. Anaerobes and fungi in chronic suppurative otitis media. Ann Otol Rhinol Laryngol. 1997;106(8):649-652.

15. Lalitha M K. Manual on Antimicrobial Susceptibility Testing (Under the auspices of Indian Association of Medical Microbiologists). 2004.

16. Clinical and Laboratory Standards Institute. Performance standards for antimicrobial susceptibility testing. Nineteenth informational supplement. Approved standard. M100-S19 Wayne, PA. 2009.

17. Acuin J. Chronic Suppurative Otitis Media. BMJ Clin Evid. 2007;88(10):694-696.

18. Bukharie HA. A review of community-acquired methicillin-resistant Staphylococcus aureus for primary care physicians. J Family Community Med. 2010;17(3):117-120.

19. Mansoor T, Musani MA, Khalid G, et al.Pseudomonas aeruginosa in chronic suppurative otitis media sensitivity spectrum against various antibiotics in Karachi.J Ayub Med Coll Abbottabad. 2009;21(2):120-123.

20. Deepak Juyal, Vikrant Negi, Munesh Shamanth Adekhandi, et al. Significance of fungal flora in C.S.O.M. Vestn Otorinolaringol. 2014;7(2):120-123.

21. Stewart PS. Mechanisms of antibiotic resistance in bacterial biofilms. Int J Med Microbiol. 2002;292(2):107-113.

22. Nadell CD, Xavier JB, Foster KR. The sociobiology of biofilms. FEMS Microbiol Rev. 2009;33(1):206-224.

23. Jana J, Susanne S, James TW. Medical biofilms: detection, prevention, and control. 2005. 